\title{
The Prognostic Significance of the Luminal A, Luminal B, Basal and Her 2 Neu Subtypes of Breast Cancer in Saudi Women
}

\author{
Eyad Fawzi Al Saeed ${ }^{1}$, Abdul Rahman Jameel Al Ghabbban² and Mutahir A. Tunio,3 \\ ${ }^{I}$ Radiation oncology, King Khalid University Hospital, King Saud University Hospital, Riyadh, Saudi Arabia \\ ${ }^{2}$ King Saud University Hospital, Saudi Arabia \\ ${ }^{3}$ Radiation Oncology, Comprehensive Cancer Center King Fahad Medical City, Riyadh, Saudi Arabia
}

\begin{abstract}
Background: Breast Cancer (BC) is molecularly diverse disease that has been sub-classified in four major subtypes; (a) luminal A [estrogen and/or progesterone positive and Her 2neu negative], (b) luminal B [estrogen and/or progesterone positive and Her 2 neu positive], (c) Basal [triple negative] and (d) Her 2 neu [estrogen and/or progesterone negative and Her 2 neu positive]. Luminal A and luminal B are less aggressive subtypes and are associated with better prognosis as compared to basal and Her 2 neu subtypes. We aimed to evaluate the pattern of breast cancer subtypes and response to treatment and outcomes in Saudi women.
\end{abstract}

Materials and Methods: Between February 1988 and August 2008, confirmed BC from a cohort of 112 patients were subtyped according to the hormone receptor status and Her 2 neu overexpression which were determined by immunohistochemistry (IHC) and Fluorescence in situ hybridization (FISH). The prognostic value of the BC subtypes for locoregional control (LRC), distant metastases control (DMC), disease free survival (DFS) and overall survival (OS) was investigated using Kaplan-Meier curves and multivariable Cox regression model.

Results: Pattern of BC subtypes in Saudi women were as; luminal A (32.1\%), Her 2 neu ((32.1\%), luminal B (25.9\%) and basal (9.8\%). Luminal A and Her 2 neu subtypes were predominant $(34.4 \%$ and $33.3 \%)$ in premenopausal and basal subtype $(26.3 \%)$ was predominant in postmenopausal women. Ten year DFS was $95 \%, 62 \%, 50 \%$ and $42.8 \%$ in luminal A, B, Her 2 neu and basal types respectively (p 0.003). No difference in LRC among different subtypes was seen ( $\mathrm{p} 0.9$ ) and DMC was found poor in Her 2 neu and basal subtypes (p 0.03).

Conclusion: Luminal A subtypes has favorable prognosis in Saudi women with breast cancer as compared to other subtypes. Molecular subtyping can be helpful in predicting the treatment outcomes in breast cancer patients.

Keywords: Breast cancer, Saudi women, biological subtypes, receptors, treatment outcomes.

\section{INTRODUCTION}

Breast cancer is the most common cancer among women in many countries including Saudi Arabia and poses a major health problem. In Saudi Arabia, there has been a steady increase in breast cancer over a decade [1]. Most of Saudi women with breast cancer are diagnosed relatively at young age and mostly have locally advanced stages and are treated with multimodality approach (neoadjuvant chemotherapy, surgery, adjuvant radiotherapy, chemotherapy and hormonal therapy) with 5 years disease free survival (DFS rates ranging from $29 \%-40 \%$ [2]. Clinical features (young age and premenopausal status), histopathological features (T stage > T3, N1-N3, absence of estrogen and progesterone receptors and overexpression of Her2 neu) have been known as major prognostic features for locoregional control (LRC), distant metastases control ((DMC) and DFS [2-7].

Molecular and gene expression studies of breast cancers have allowed the additional classification of breast cancers

*Address correspondence to this author at the King Fahad Medical City, Riyadh, Saudi Arabia; Tel: +96612889999; Fax: +96612889999;

E-mail: drmutahirtonio@hotmail.com according to molecular subtypes; (a) luminal A [estrogen and/or progesterone positive and Her 2neu negative], (b) luminal B [estrogen and/or progesterone positive and Her 2 neu positive], (c) Basal [triple negative] and (d) Her 2 neu [estrogen and/or progesterone negative and Her 2 neu positive] [8]. Patients with basal subtypes are known to have the worst overall survival, reflected by the abundance of triple negative (ER-negative, PR-negative and ERBB2negative) tumors followed by patients with cancer subtypes of Her 2 neu [9]. Both these subtypes are also more sensitive to neoadjuvant chemotherapy than luminal A and B subtypes [10]. In addition, among the luminal subtype of tumors, the luminal B subtype has been found less responsive to hormonal therapy and has a less favorable treatment outcome as compared to luminal A subtype [11].

However molecular classification and its prognostic significance in Saudi women with breast cancers have not been studied. We aimed to evaluate the frequency of these molecular subtypes and their impact on treatment outcomes in Saudi women with breast cancer. 


\section{MATERIALS AND METHODS}

After the approval from Institutional Ethical Review Board (IRB) committee, 112 patients with breast cancer were treated between February 1988 and August 2008 with BCS and MRM followed by adjuvant chemotherapy and radiotherapy and comprised the study population. Inclusion criteria were; (a) histopathological confirmed breast cancer, (b) T1-T4, N0-N2, (c) underwent BCS or MRM +/- adjuvant hormonal, chemotherapy and radiotherapy. Exclusion criteria were; (a) presence of distant metastasis, (b) neoadjuvant chemotherapy and (c) inflammatory or inoperable tumors.

\section{Breast Cancer Pathological Specimens}

After surgery, breast cancer specimens were fixed in 10\% formalin overnight and were examined to evaluate the, gross size, histopathological type, grade, presence or absence of lymphovascular (LVSI) invasion, lymph nodes and retrieved and involved number. Immunohistochemical (IHC) analysis was performed to classify the breast cancers according to IHC-based breast cancer subtypes and also fluorescence in situ hybridization (FISH) was performed.

\section{Molecular Sub-Typing}

Estrogen receptors (ER), progesterone receptors (PgR) and Her 2 neu overexpression status was performed by IHC analysis with antibodies against the ER (Dako, Denmark) and PR (BioGenex, San Ramon, CA). Cut off value of $1 \%$ for both receptors was considered ER or PR presence as per to institutional protocol [7].

Her 2 neu overexpression was checked for IHC equivocal cases using a HER2 DNA Probe Kit (Abbott) according to the manual instructions. Her 2 neu was considered positive when the HER2/CEP 17 ratio was 2.1 or higher [10].

Finally, breast cancers were categorized into four subtypes as; (a) luminal A [estrogen and/or progesterone positive and Her 2 neu negative], (b) luminal B [estrogen and/or progesterone positive and Her 2 neu positive], (c) Basal [triple negative] and (d) Her 2 neu [estrogen and/or progesterone negative and Her 2 neu positive].

\section{Clinical Variables}

Additional clinical features including age, menopausal status, associated co-morbidities, initial tumor size on physical, mammography and sonography examination, laterality and location of primary tumor were studied. Surgery was performed either wide local excision or MRM with axillary lymph node dissection.

\section{Statistical Analysis}

The primary endpoints were frequency of molecular subtypes, correlation with clinical and histopathological features, locoregional control (LRC), distant metastases control (DMC), disease free survival (DFS) and overall survival (OS) according to breast cancer subtypes. The times to last follow up evaluation, appearance of local relapse and death were calculated from date of starting treatment. DFS was defined as the duration between the entry date and the date of documented disease reappearance, death from cancer and/or last follow-up (censored). OS was defined as the duration between the entry date and the date of patient death or last follow-up (censored). Probabilities of LRC and DMC, DFS and OS were determined with the Kaplan-Meier method. The comparisons between various endpoints were performed using log rank test and Cox proportional hazard analysis. Univariate and multivariate analyses were also performed for different prognostic factors for LRC, DMC, DFS and OS. All statistical analyses were performed using the computer program SPSS version 16.0.

\section{RESULTS}

Median follow up period was 9 years (range: 5-17). Patients' characteristics according to molecular breast cancer subtypes were shown in Table 1. Predominant breast cancer subtypes were luminal A (36 patients; $32.2 \%$ ) and Her2 neu (36 patients; $32.2 \%$ ). Basal like subtype was least frequent (11 patients; $9.8 \%$ ).

\section{Clinical Characteristics}

Mean age of cohort was 47.0 years \{range: 23-76; standard deviation (SD) 10.3. According to menopausal status, 93 patients $(83.0 \%)$ were premenopausal and 19 patients (17.0\%) were postmenopausal. Mean BMI was 31.8 $\mathrm{kg} / \mathrm{m}^{2}$ (range: 15.7-52.8; SD7.2). According to comorbidities, 72 patients $(64.3 \%)$ had no co-morbidities. Common morbidities in 40 patients (35.7\%) were; hypertension in 14 patients $(12.5 \%)$, diabetes in 9 patients $(8.0 \%)$ and combined hypertension and diabetes in 6 patients $(5.4 \%)$. Family history was positive in 17 patients $(15.2 \%)$. In four patients $(3.6 \%)$ there was bilateral breast cancer at the time of diagnosis. Majority of cohort (57 patients; $79.6 \%$ ) had left side breast cancer and outer lower quadrant was common site of tumor location (50 patients; $45.9 \%$ ) followed upper outer quadrant (30 patients; $27.5 \%$ ). Mean mammography size of tumor was $3.9 \mathrm{~cm}$ (range: 1-7; SD 2.3)). Mean baseline CA15.3 level was 31.1 units/ml (range: 1-94.3; SD 23.9).

\section{Histopathological Characteristics}

Mean pathological tumor size was $3.4 \mathrm{~cm}$ (range: 1-10; SD 2.4) and infiltrating ductal carcinoma (IDC) was predominant histopathological variant seen in 95 patients $(88.0 \%)$. Majority of tumors were moderately differentiated (GII) (68 patients; 60.7\%). According to marginal status, 77 patients $(68.8 \%)$ had negative margins.

\section{Treatment Characteristics}

Majority of patients had MRM (77 patients; 71.3\%) followed by BCS in 31 patients (28.7\%). Mean axillary retrieved lymph nodes were 14.1 (2-42) after axillary dissection and sentinel node dissection.

Total 70 patients $(62.5 \%)$ received adjuvant chemotherapy which was given within 5-6 weeks (range: 311) of surgery. Adjuvant chemotherapy protocols were as; CMF (18.6\%), AC followed Paclitaxal (32.9\%), FAC (32.9\%), TAC (5.9\%), AT (5.7\%), FAC+TAC (4\%).

Total 89 patients $(83.0 \%)$ received local radiation therapy which was started 22 weeks (18-26) after the surgery and 3-5 weeks after the completion of chemotherapy. For BCS patients, whole breast radiation therapy was delivered in $2 \mathrm{~Gy}$ fractions to mean total mean dose of $50 \mathrm{~Gy}$ (45-64) and for 
Table 1. Patients Characteristics According to Molecular Subtypes Groups

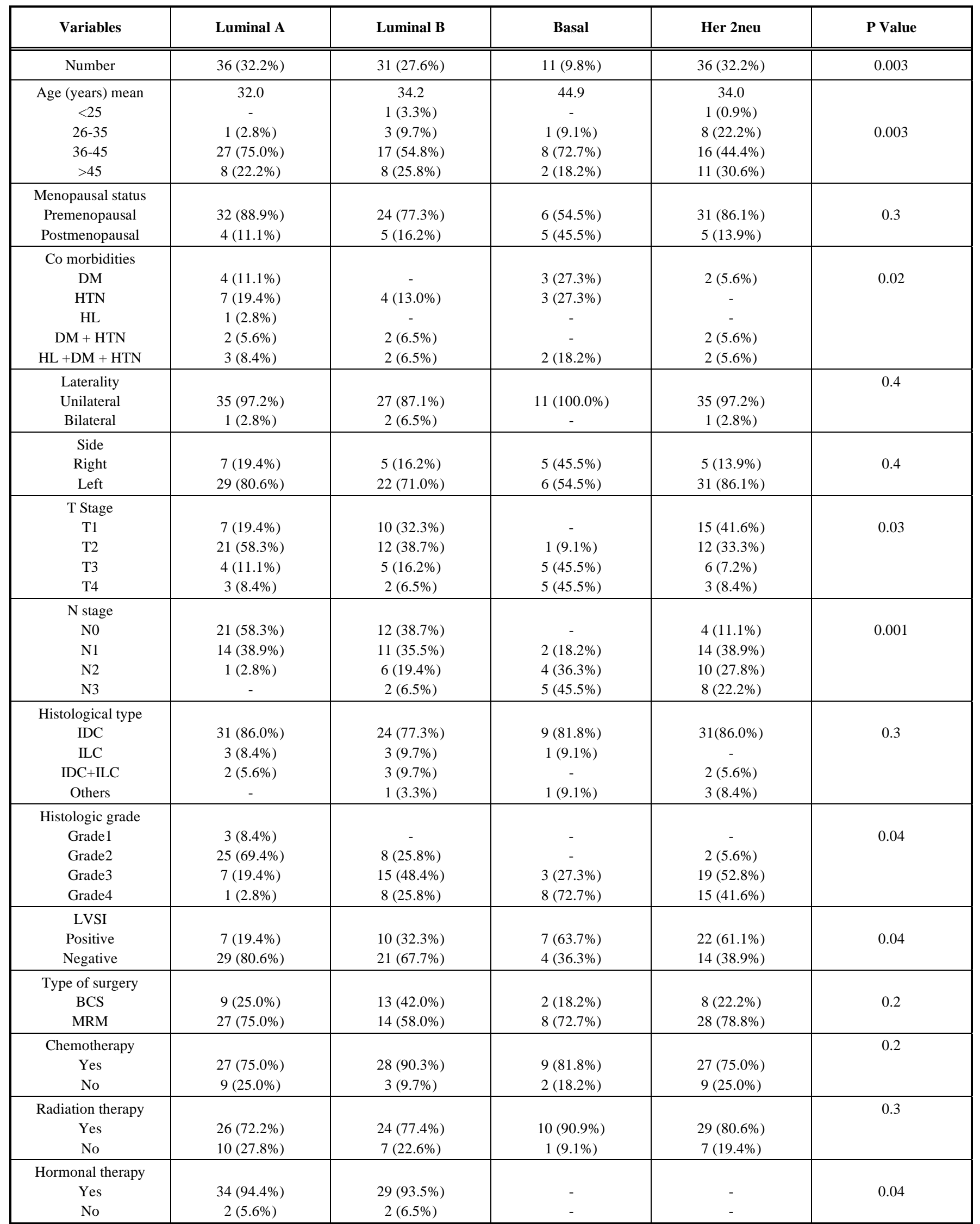

Abbreviations: DM= diabetes mellitus, HTN= hypertension, HL= hyperlipidemia, T=Tumor, N=Nodes, IDC=infiltrating ductal carcinoma, ILC= invasive lobular carcinoma, LVSI= lymphovascular space invasion, $\mathrm{BCS}=$ breast conservation surgery, MRM=modified radical mastectomy, 
MRM patients, chest wall radiation therapy was given in 2 Gy fractions to mean total dose 50Gy (50-60). For positive axillary nodes, supra-clavicular fields were used and given mean total dose of $45 \mathrm{~Gy}$ (42.5-50). All patients were treated using three dimensional conformal radiation therapy (3DCRT) and on multileaf collimator (MLC) assisted linear accelerator.

Sixty three patients were given hormonal therapy in form of tamoxifen (46.4\%), letrozole $(9.8 \%)$ and sequential regimen in $9.8 \%$. Mean duration of hormonal therapy was 5.5 years (range; $3.5-7.2$ ).

\section{Locoregional Recurrence, Distant Control and Overall Survival}

Total ten locoregional recurrences $(8.93 \%)$ were seen among 112 breast cancer patients. Four (40\%) LRR occurred at scar site and 6 LRR $((60 \%)$ were seen in ipsilateral axilla and supraclavicular regions. Median time of LRR was 3.6 years (range: 2.73-5.1). The actual LRC rates at 5 and 10 years were $86.4 \%$ (Fig. 1). Distant metastases were documented in 29 patients at 5 years and 37 patients at 5 and 10 years with 5 and 10 actual DMC rates of $63 \%$ (95\% CI: 57-79.0) and 56.7\% (95\% CI:54 - 65.2) respectively (Fig. 2). Actual 5 and 10 years DFS rates were $77.4 \%$ and $66.4 \%$ respectively (Fig. 3). Five and 10 years OS rates were $74.2 \%$ and $68.7 \%$ respectively. According to breast cancer subtypes, there was no difference between LRR, however luminal A subtypes had better DMC and DFS rates and Basal like subtype was related to poor DMC and DFS (Figs. 4-6).

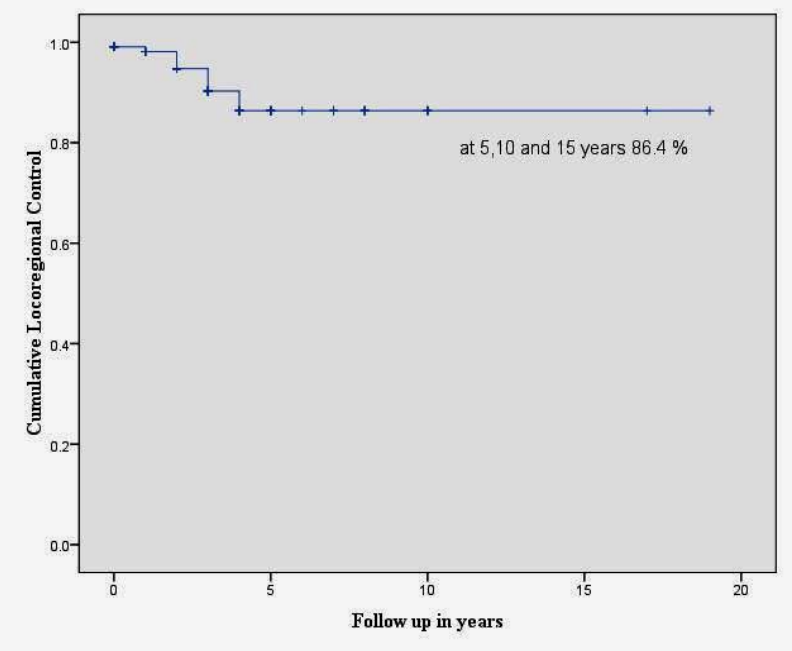

Fig. (1). Cumulative locoregional control in study cohort.

Cox proportional analysis of clinical and histopathological prognostic factors in our cohort showed five prognostic factors influencing the LRR; (a) age less than 45, (b) premenopausal status, (c) presence of LVSI and (d) no adjuvant radiotherapy (Table $\mathbf{2 A}$ ). For DMC, in addition to age less than 45 years and premenopausal status, breast cancer subtypes were found important prognostic factors (Table 2B, 2C).

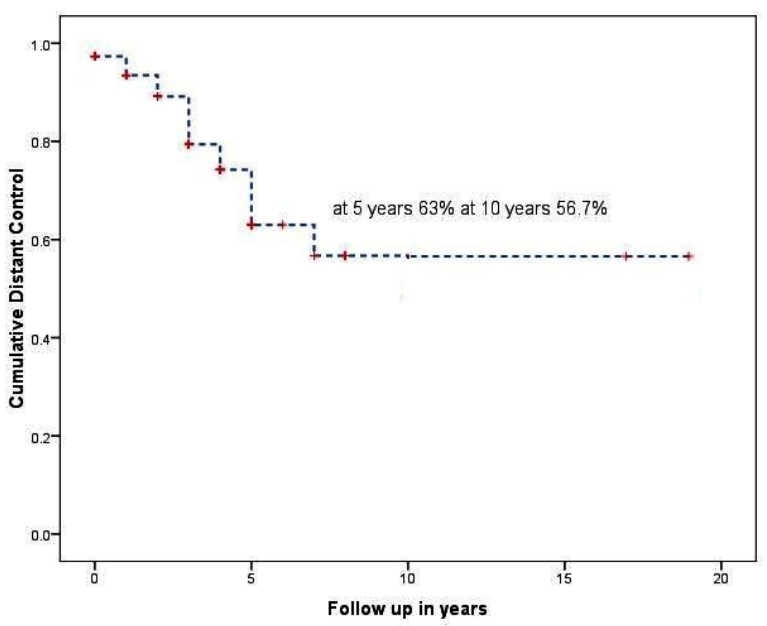

Fig. (2). Cumulative distant metastases control in study cohort.

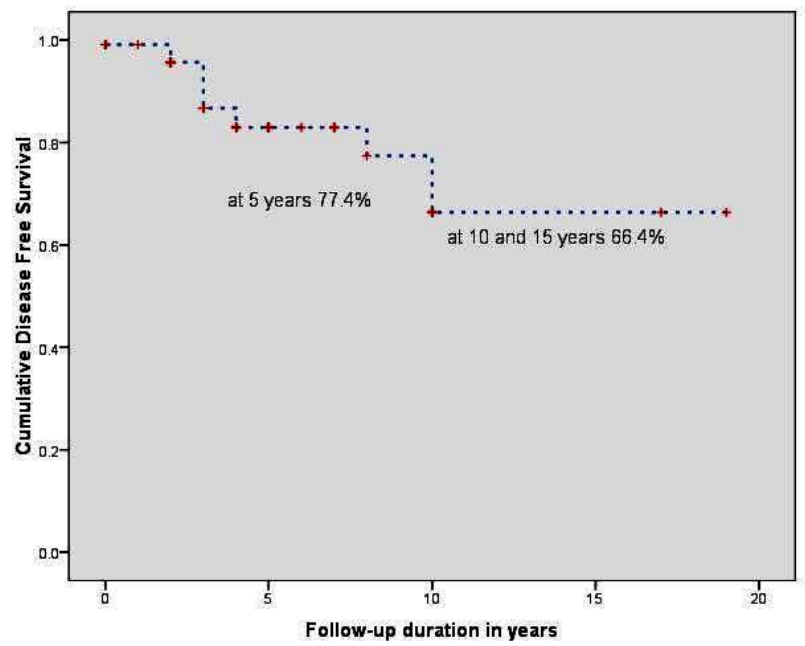

Fig. (3). Cumulative disease free survival in study cohort.

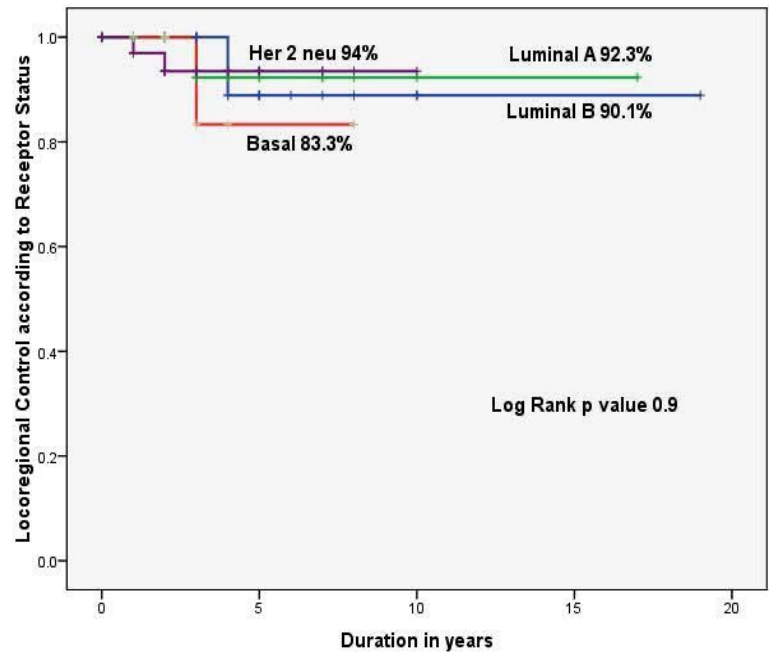

Fig. (4). Locoregional control according to breast cancer subtypes. 
Table 2. (A) Multivariate Analysis of Prognostic Factors for Locoregional Recurrence in Breast Cancer

\begin{tabular}{|c|c|c|c|}
\hline Variables & HR & Confidence Intervals & P Value \\
\hline \hline Age $<45 v s>45$ & 2.7 & $2.2-3.1$ & $\mathbf{0 . 0 2}$ \\
\hline Premenopausal $v s$ postmenopausal & 2.4 & $1.9-2.9$ & 0.03 \\
\hline Luminal A $v$ s luminal B & 1.2 & $0.9-1.3$ & 0.05 \\
\hline Luminal A $v s$ other subtypes & 1.1 & $0.8-1.2$ & 0.4 \\
\hline$<$ T3 $v s$ T4 & 2.2 & $1.7-3.2$ & $\mathbf{0 . 0 1}$ \\
\hline N1 $v s$ N2 & 2.8 & $2.1-3.9$ & 0.001 \\
\hline$<$ G3 $v s>$ G3 & 1.1 & $0.8-1.2$ & 0.5 \\
\hline LVSI (+) $v s$ LVSI (-) & 1.7 & $1.2-2.3$ & $\mathbf{0 . 0 2}$ \\
\hline Adjuvant RT $v s$ no RT & 3.8 & $2.7-4.2$ & 0.001 \\
\hline Adjuvant CT $v s$ no CT & 1.1 & $0.7-1.2$ & 0.5 \\
\hline Adjuvant HT $v s$ no HT & 1.1 & $0.7-1.2$ & 0.5 \\
\hline
\end{tabular}

(B) Multivariate Analysis of Prognostic Factors for Distant Metastasis in Breast Cancer

\begin{tabular}{|c|c|c|c|}
\hline Variables & HR & Confidence Intervals & P Value \\
\hline \hline Age $<45 v s>45$ & 2.6 & $2.1-3.0$ & $\mathbf{0 . 0 2}$ \\
\hline Premenopausal $v s$ postmenopausal & 1.4 & $1.2-1.9$ & 0.04 \\
\hline Luminal A $v s$ luminal B & 5.2 & $3.7-7.3$ & $\mathbf{0 . 0 0 0 1}$ \\
\hline Luminal A $v s$ other subtypes & 4.7 & $3.6-7.2$ & $\mathbf{0 . 0 0 0 1}$ \\
\hline$<$ T3 $v s$ T4 & 2.0 & $1.5-2.5$ & $\mathbf{0 . 0 1}$ \\
\hline N1 $v s$ N2 & 3.9 & $2.6-4.2$ & 0.001 \\
\hline S G3 $v s>$ G3 & 1.1 & $0.8-1.2$ & 0.5 \\
\hline Preoperative CA15.3 $35 v s>35$ & 3.8 & $2.5-4.1$ & 0.001 \\
\hline LVSI (+) $v s$ LVSI (-) & 1.1 & $0.8-1.2$ & 0.5 \\
\hline Adjuvant RT $v s$ no RT & 1.2 & $1.0-1.3$ & 0.3 \\
\hline Adjuvant CT $v s$ no CT & 5.2 & $4.7-8.2$ & $\mathbf{0 . 0 0 0 1}$ \\
\hline Adjuvant HT $v s$ no HT & 3.1 & $2.6-5.2$ & $\mathbf{0 . 0 0 1}$ \\
\hline
\end{tabular}

(C) Multivariate Analysis of Prognostic Factors for Disease Free Survival in Breast Cancer

\begin{tabular}{|c|c|c|c|}
\hline Variables & HR & Confidence Intervals & P Value \\
\hline \hline Age $<45 v s>45$ & 2.5 & $2.0-2.9$ & $\mathbf{0 . 0 2}$ \\
\hline Premenopausal $v s$ postmenopausal & 1.4 & $1.2-1.9$ & 0.04 \\
\hline Luminal A $v s$ luminal B & 4.3 & $3.6-6.2$ & $\mathbf{0 . 0 0 1}$ \\
\hline Luminal A $v s$ other subtypes & 5.7 & $4.5-8.2$ & $\mathbf{0 . 0 0 0 1}$ \\
\hline$<$ T3 $v s$ T4 & 2.0 & $1.5-2.5$ & $\mathbf{0 . 0 1}$ \\
\hline N1 $v$ N2 & 2.9 & $2.5-3.5$ & 0.002 \\
\hline$<$ G3 $v s>$ G3 & 1.1 & $0.8-1.2$ & 0.5 \\
\hline LVSI (+) $v s$ LVSI (-) & 1.1 & $0.8-1.2$ & 0.5 \\
\hline Preoperative CA15.3 $<35 v s>35$ & 2.7 & $2.4-2.9$ & $\mathbf{0 . 0 0 2}$ \\
\hline Adjuvant RT $v s$ no RT & 1.1 & $0.9-1.2$ & 0.3 \\
\hline Adjuvant CT $v s$ no CT & 3.5 & $3.0-5.2$ & $\mathbf{0 . 0 0 0 1}$ \\
\hline
\end{tabular}




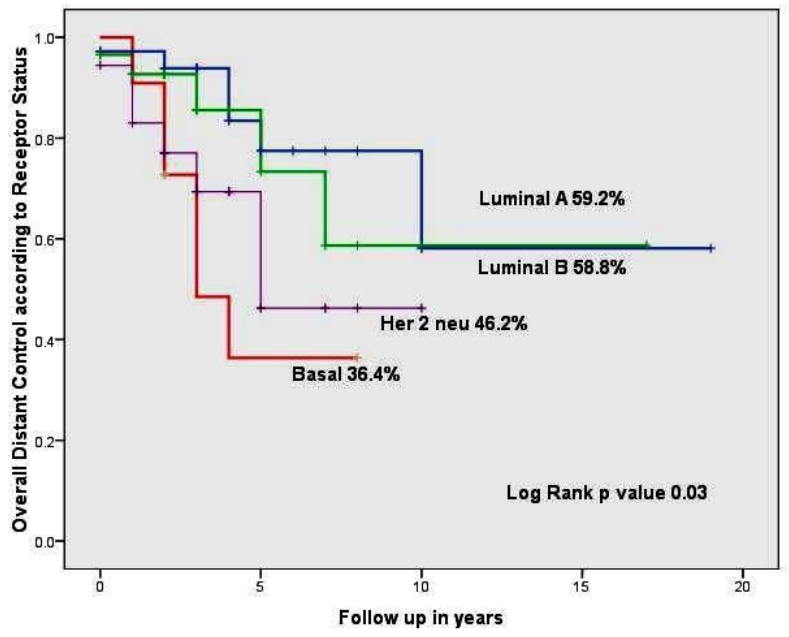

Fig. (5). Distant metastases control according to breast cancer subtypes.

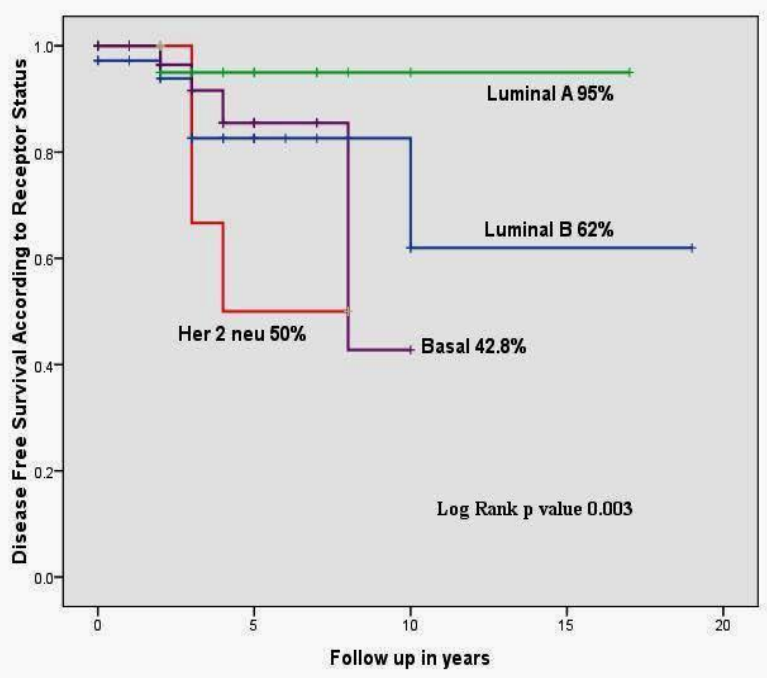

Fig. (6). Disease free survival according to breast cancer subtypes.

\section{DISCUSSION}

In past decade, a molecular classification system was proposed to divide breast cancers into subtypes owing to response to optimal treatment and it has become widely used these days [12]. However molecular classification has not been studied in Saudi women with breast cancer. In present study, the pattern of breast cancer subtypes, association with clinical and histopathological features and LRR, DMC, DFS and OS according to breast cancer subtypes in Saudi women were studied.

Key findings in present study were;

a. There was similarity in clinical and histopathological features of luminal A and luminal B subtypes but difference in DFS rates $(33 \%$ increased DFS associated with luminal A), which is consistent with results of other similar studies [13, 14].

b. Her 2 neu over-expression was seen in $32.2 \%$, which is much higher than reported by western studies [15]. c. Basal like subtype was least frequent but associated with worst DMC and DFS rates as compared to other subtypes which are consistent with results of reported literature [16]. However, in terms of response to chemotherapy, many studies in which breast cancer patients were treated with neoadjuvant anthracyclinebased chemotherapy have shown higher response rates in basal subtypes as compared to luminal subtypes [10, 17]. Adjuvant non anthracycline-based chemotherapy had minimal impact on basal subtypes [18].

d. Breast cancer subtypes were not found significant prognostic factor for LRC rates.

Our study can criticized for (a) low sample size, (b) Ki67 index was not performed to assess tumor cell proliferation [19] and (c) lack of DNA ploidy assessment, which have been known as important prognostic factors for DMC and DFS rates [20], which shall be addressed in future studies of Saudi women with breast cancer.

In conclusion, frequency pattern, clinicopathological characteristics and treatment outcomes of breast cancer subtypes in Saudi women are consistent with other related studies; however the Her 2 neu overexpression is more in Saudi women. Breast cancer subtype classification may be helpful if considering neoadjuvant chemotherapy (basal subtype more responsive) and hormonal therapy (luminal B less responsive).

$\begin{array}{lll}\text { ABBREVIATIONS } \\ \mathrm{BC} & = & \text { Breast Cancer } \\ \mathrm{BCS} & = & \text { Breast conserving surgery } \\ \mathrm{DFS} & = & \text { Distant metastases control } \\ \mathrm{DMC} & = & \text { Fluorescent In Situ hybridization } \\ \mathrm{FISH} & = & \text { Immunohistochemistry } \\ \mathrm{IHC} & = & \text { Locoregional control } \\ \mathrm{LRC} & = & \text { Locoregional recurrence } \\ \mathrm{LRR} & = & \text { Modified radical mastectomy } \\ \mathrm{MRM} & = & \text { Overall survival }\end{array}$

\section{CONFLICT OF INTEREST}

The authors confirm that this article content has no conflict of interest.

\section{ACKNOWLEDGEMENTS}

Declared none.

\section{REFERENCES}

[1] Elkum N, Dermime S, Ajarim D, et al. Being 40 or younger is an independent risk factor for relapse in operable breast cancer patients: the Saudi Arabian experience. BMC Cancer 2007; 7: 22.

[2] Ezzat AA, Ibrahim EM, Raja M, et al. Locally advanced breast cancer in Saudi Arabia: high frequency of stage III in a young population. Med Oncol 1999; 16: 95-103.

[3] Uhrhammer $\mathrm{N}$, Abdelouahab A, Lafarge L, et al. BRCA 1 mutations in Algerian breast cancer patients: high frequency in young, sporadic cases. Int J Med Sci 2008; 5: 197-202.

[4] Al-Idrissi HY, Ibrahim EM, Kurashi NY, Sowayan SA. Breast cancer in a low- risk population. The influence of age and 
menstrual status on disease pattern and survival in Saudi Arabia. Int J Cancer 1992; 52: 48-51.

[5] Ibrahim EM, Ezzat AA, Rahal MM. Adjuvant chemotherapy in 780 patients with early breast cancer: 10-year data From Saudi Arabia. Med Oncol 2005; 22: 343-52.

[6] Al Saad SK, Jalal AA. Breast cancer risk factors and stage at presentation. Bahrain Med Bull 2006; 28: 111-5.

[7] Paszko Z, Omar YT, Nasralla MY, et al. Estrogen and progesterone receptor status in breast cancer in Kuwait female population. Neoplasma 1993; 40: 127-32.

[8] Sotiriou C, Neo SY, McShane LM, et al. Breast cancer classification and prognosis based on gene expression profiles from a population-based study. Proc Natl Acad Sci USA 2003; 100: 10393-8.

[9] Hu Z, Fan C, Oh DS, et al. The molecular portraits of breast tumors are conserved across microarray platforms. BMC genomics 2006; 7: 96.

[10] Rouzier R, Perou CM, Symmans, et al. Breast cancer molecular subtypes respond differently to preoperative chemotherapy. Clin Cancer Res 2005; 11: 5678-85.

[11] Raica M, Jung I, Cimpean AM, et al. From conventional pathologic diagnosis to the molecular classification of breast carcinoma: are we ready for the change? Rom J Morphol Embryol 2009; 50: 5-13.

[12] Goldhirsch A, Wood WC, Coates AS, et al. Panel members. Strategies for subtypes--dealing with the diversity of breast cancer: highlights of the St. Gallen international expert consensus on the primary therapy of early breast cancer 2011. Ann Oncol 2011; 22: $1736-47$.
[13] Penault-Llorca F, André F, Sagan C, et al. Ki67 expression and docetaxel efficacy in patients with estrogen receptor-positive breast cancer. J Clin Oncol 2009; 27: 2809-15.

[14] Colleoni M, Bagnardi V, Rotmensz N, et al. A nomogram based on the expression of $\mathrm{Ki}-67$, steroid hormone receptors status and number of chemotherapy courses to predict pathological complete remission after preoperative chemotherapy for breast cancer. Eur $\mathbf{J}$ Cancer 2010; 46: 2216-24.

[15] Fitzgibbons PL, Page DL, Weaver D, et al. Prognostic factors in breast cancer. College of American Pathologists Consensus Statement 1999. Arch Pathol Lab Med 2000; 124: 966-78.

[16] Cheang MC, Voduc D, Bajdik C, et al. Basal-like breast cancer defined by five biomarkers has superior prognostic value than triple-negative phenotype. Clin Cancer Res 2008; 14: 1368-76.

[17] Carey LA, Dees EC, Sawyer L, et al. The triple negative paradox: primary tumor chemosensitivity of breast cancer subtypes. Clin Cancer Res 2007; 13: 2329-34.

[18] Conforti R. Breast cancer molecular subclassification and estrogen receptor expression to predict efficacy of adjuvant anthracyclinesbased chemotherapy: a biomarker study from two randomized trials. Ann Oncol 2007; 18: 1477-83.

[19] Cheang MC, Chia SK, Voduc D, et al. Ki67 index, HER2 status, and prognosis of patients with luminal B breast cancer. J Nat Cancer Inst 2009; 101: 736-50.

[20] Yildirim Assaf S, Coumbos A, Hopfenmüller W, et al. The prognostic significance of determining DNA content in breast cancer by DNA image cytometry: the role of high grade aneuploidy in node negative breast cancer. J Clin Pathol 2007; 60: 649-55.

(C) AlSaeed et al.; Licensee Bentham Open.

This is an open access article licensed under the terms of the Creative Commons Attribution Non-Commercial License (http://creativecommons.org/licenses/by-nc/ $3.0 /$ ) which permits unrestricted, non-commercial use, distribution and reproduction in any medium, provided the work is properly cited. 